\title{
A Wearable Fabric Computer by Planar-Fashionable Circuit Board Technique
}

\author{
Hyejung Kim, Yongsang Kim, Binhee Kim, and Hoi-Jun Yoo \\ Electrical Engineering, KAIST \\ Daejeon, Republic of Korea \\ seeseah@eeinfo.kaist.ac.kr
}

\begin{abstract}
A method to fabricate circuits on the cloth, planar fashionable circuit board (P-FCB), is proposed. And its applications such as fabric passive elements, user $\mathrm{I} / \mathrm{O}$ interface, and the fabric package are introduced. The electrical and the mechanical characteristic analysis of P-FCB and the system integration methodology establishment improve the system performance and productivity. A complete wearable system is implemented by P-FCB technology for the continuous sweat monitoring and the RFID tag antenna applications.
\end{abstract}

Keywords- Fabric circuit board, capacitive sensor, sweat monitoring

\section{INTRODUCTION}

Recently, with the increase of the interests about the mobile and the wearable system, e-textile is drawn the attention. The e-textile means that the computing and communication functions are embedded into the textile itself. The Pervasive features such as flexibility, low cost, robustness, unobtrusiveness, washability, small size, biocompatibility with human skin, and aesthetic sense are important requirement for the e-textile.

Many researches have been reported on the integration of electronics with textiles such as the wearable computer and e-textiles [1]-[9]. At first, the wearable computer consists of bulky electric device attachment on the clothes [3]. Since those wearable computers are big and heavy to carry on, the e-textile is proposed. For the e-textile, devices should be developed to be as soft, flexible and comfortable as conventional clothes. Most of the previous e-textile studies were based mainly on the conducting threads, yarns, and woven fabrics for inter-connections like wearable motherboard [4]. For the integration of IC with fabric, they placed the IC on a plastic package or a flexible PCB, and then integrated on the fabric [1-9]. However, the plastic board is harder than fabric and may generate discomfort feeling in the clothes. A new concept of e-textile is necessary for more pervasiveness and flexibility.

In this paper, we apply the planar thin/thick film technology on the fabric which is named as "planar fashionable circuit board' (P-FCB) [1]. Since the fabric is selected as a substrate, the P-FCB has the same mechanical properties as the clothes. The P-FCB can be easily processed into the various clothes such as clothes, a bag, and a pillow as a normal textile and the electronic devices such as planar fabric sensors, LED display, fabric chip package.

\section{PLANAR - FASHIONABLE CIRCUIT BOARD}

\section{A. P-FCB Manufacturing Process}

The P-FCB substrate is fabricated on the casual woven fabrics by silk screening of conducting epoxy and by gold sputtering. The planar electrodes are deposited directly on the fabric patch. The fabric substrate serves flexibility, durability and light weight. The proposed planar circuit printing approaches can make entire electronic circuits on a fabric board at once and also reproduce the identical circuit boards repeatedly, resulting in low cost, high productivity and high quality circuit board, just as the electronic PCB technology does.

The silk screening process is a simple and low cost process which is well established in electronics industry to make complicated electronic circuitry on the plastic boards [10]. The proposed technology, however, uses a conductive ink to form the electronic circuits rather than to use simple colored ink to paint figures on the clothes. The conductive paste consisting of silver, polymer, solvent, polyester and cyclohexa- none is used as the silk screen ink due to its high conductivity and adhesiveness to the fabrics. The printing process is performed at room temperature. The minimum line resolution by silk screening is $200 \mu \mathrm{m}$ and the thickness is $10 \mu \mathrm{m}$.

Sputtering process can be used to form the high resolution circuits on the fabric. The gold $(\mathrm{Au})$ target is used for the sputtering with Ar inert gas plasma, and the fabric is rolled up on the roller in the vacuum chamber. A shadow mask with the circuit patterns formed in it is placed on the fabric, and $\mathrm{Au}$ atoms are sputtered on the fabric passing

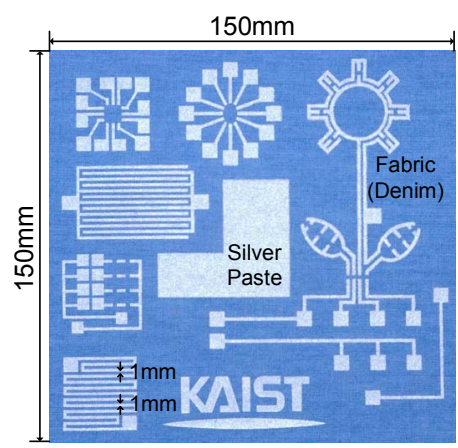

(a) Silk Printing

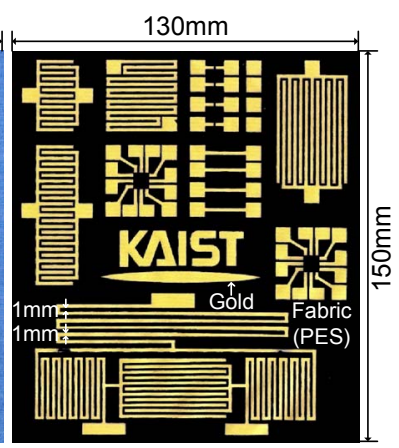

(b) Sputtering
Figure 1. atterned Fabric Circuit Board 
through the mask to form the electrical circuits. The vacuum level is $10^{-3}$ torr and the substrate temperature is $150^{\circ} \mathrm{C}$. The minimum line width of the sputtered patterns is $100 \mu \mathrm{m}$ and the thickness is $1 \mu \mathrm{m}$. Sputtering process provides electrically rigid connection and good washing endurance, but when compared with screen printing, its process is relatively expensive and time consuming.

The various fabrics are tested for the possible substrates of the P-FCB process. The fabrics are made of cotton, viscose, silk, wool, polyester (PES), polyacrylonitrile (PAN). To reduce the resistance and to maintain the stability of connection, the dense fabric with thin yarn is better. Among the fabrics, the PES is the best selection because of densest fabric structure and the small elasticity.

\section{B. Characteristic of $P-F C B$}

The characteristic of $\mathrm{P}-\mathrm{FCB}$ such as DC resistance, AC impedance, and maximal signal frequency are investigated by time-domain reflectometry (TDR) profile and frequency characterization [2]. Table 1 summarizes the characteristic of P-FCB.

DC Resistance : The DC resistance of the film wire on fabric with width of $1 \mathrm{~mm}$ and thickness of $20 \mu \mathrm{m}$ is measured as $13.35 \Omega / \mathrm{m}$ by the four-point probe method. Like the general resistive wires, the DC resistance of the $\mathrm{P}-\mathrm{FCB}$ is proportional to the length and inversely proportional to its width and thickness.

AC Impedance : The measured AC impedance value is about 201.8 $\Omega$. However, impedance variation along the transmission line is more critical rather than the absolute value. The variation of $\mathrm{AC}$ impedance is measured under $13.1 \%$.

Frequency Characteristic : S21 parameters for $15 \mathrm{~cm}$ long lines are measured with vector network analyzer (VNA) up to $3 \mathrm{GHz}$ to investigate the frequency characteristics of the transmission lines. The bandwidth of the line is measured as $80 \mathrm{MHz}$.

Tensile Strength : For the robustness from the external disturbance, the tensile strength test is performed by straining the $\mathrm{P}-\mathrm{FCB}$ along the $\mathrm{x}$-direction, y-direction, and the diagonal direction. The maximum load is $85.1 \mathrm{~N} / \mathrm{cm}$ in the $\mathrm{x}$ and $\mathrm{y}$ directions, and $99.3 \mathrm{~N} / \mathrm{cm}$ in the diagonal direction.

TABLE I. SUMMARY TABLE OF CHARACTERISTIC OF P-FCB

\begin{tabular}{|l|l|l|}
\hline \multicolumn{2}{|c|}{ Characteristic } & \multicolumn{1}{c|}{ Value } \\
\hline \hline DC Resistance & $13.35 \Omega / \mathrm{m}$ \\
\hline \multirow{2}{*}{ AC Impedance } & Value & $201.8 \Omega$ \\
\cline { 2 - 3 } & Variance & $<13 \%$ \\
\hline Bandwidth & $80 \mathrm{MHz}$ \\
\hline Wash Test & $>50$ \\
\hline
\end{tabular}

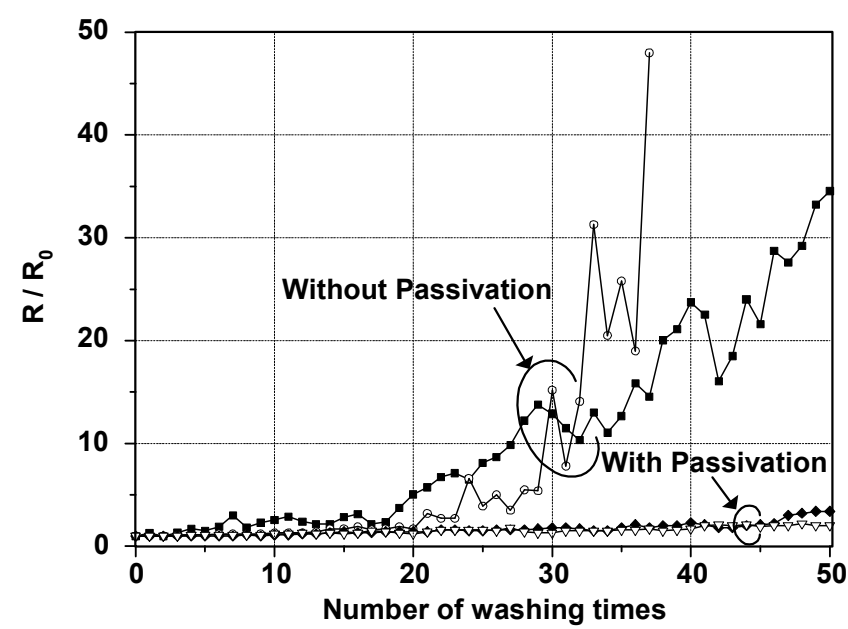

Figure 2. Variance of Resistance With Washing

Washability : In wearable computer applications, the washing endurance is very important to integrate electronic devices onto real clothes. Coating of the passivation materials, for example, polyurethane, can improve the endurance of the P-FCB against chemical and mechanical attacks like washing. Several tens of washing tests including dry cleaning and water-washing with the detergent are performed to the P-FCB fabrics. The samples without passivation experience thinning of its wire thickness or even the open connections, and their resistance values increase up to 40 times of its original value after about 20 washes. With the passivation material coated, their endurance against washings increases up, and the variation of the resistance values is less than 2 times of their original values after 50 washes as shown in Fig.2.

\section{ELECTRONIC ELEMENTS BY P-FCB PROCESS}

Although the conventional elements can be integrated on the fabric by soldering, they have the possibility of coming off from the fabric. Therefore, we propose pervasive fabric electronic elements by P-FCB. The fabric electronic element has the same properties as the conventional devices and the fabric.

\section{A. Passive Elements : $R, L$, and $C$}

Capacitor : The fabric capacitor is fabricated with interdigitated electrode of $1 \mathrm{~mm}$ line width and spacing (Fig.3(a)). Its capacitance value can be changed by dielectric constant of the fabric, and the number of interdigitated electrode controls the sensitivity.

Resistor : The fabric resistors can be implemented by long wires. A meandering wire of $30 \mathrm{~mm}$ with $1 \mathrm{~mm}$ width and space (Fig.3(b)) behaves as a resistor of $85 \Omega$. The measured resistivity of the film wire is larger than the conductive paste due to the non-uniformity of the surface and the corners of the meandering wire. 
Inductor : Fig.3(c) show a spiral inductor on P-FCB and their measured inductance values are about $970 \mathrm{nH}$ with $\mathrm{Q}=6.0$ at $10 \mathrm{MHz}$. Measured $\mathrm{Q}$ factors are quite small due to their planar spiral structures and large resistances. The fabric inductor can be used for an antenna or filter. The value of the passive element can be varied by changing a shape, a type of material, a type of substrate textile, and a thickness of silver paste.

\section{B. Fabric Packag}

Most of conventional wearable systems have used the plastic packaged modules which have large size and hard package. In this work, the bare die is directly integrated into the clothes. The fabric package contributes to the system size, wearability, and simple user interface. Fig.4 shows the process of the fabric package fabrication. First, the lead frames are printed on the fabric (Fig.4(a)). Since the limitation of lead width and space, maximum 16 pads are allowed in the $25 \times 25 \mathrm{~mm}^{2}$ patch. The chip is attached on the lead frame, then gold wire is bonded on the pads of the chip and the lead frame on the P-FCB (Fig.4(b)). Of course the flip-chip bonding can be used on P-FCB [2]. After the bonding process, liquid molding epoxy is coated on the exposed electrodes. This molding process provides highly robust protection against potential pressure on the packaged chip (Fig.4(c)).

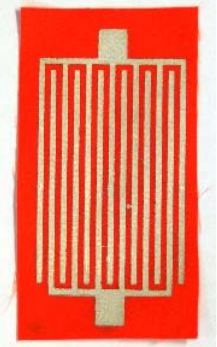

(a) Capacitor

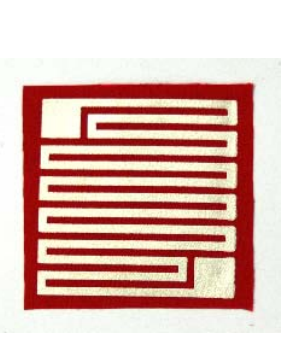

(b) Resistor

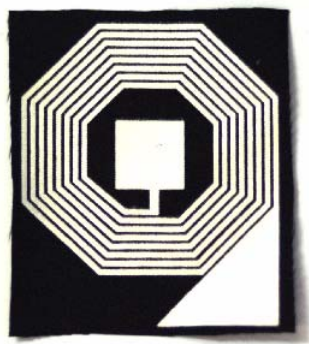

(c) Inductor
Figure 3. Passive Elements by P-FCB Technology

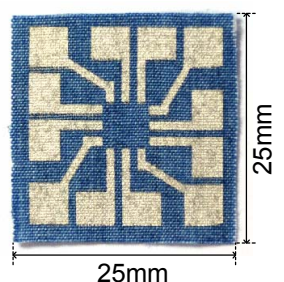

(a) Lead Frame

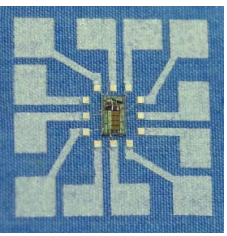

(b) Wire Bonding (c) Fabric Chip Package

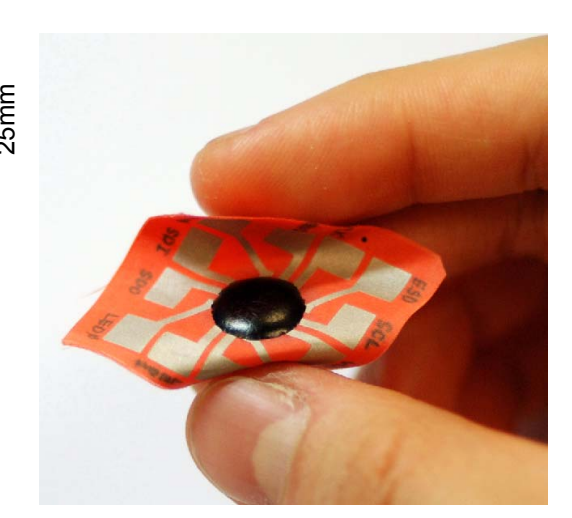

Figure 4. Process of Fabric Package

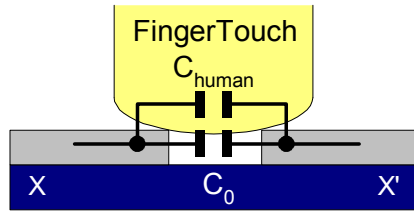

(a) Fabric Touch Sensor

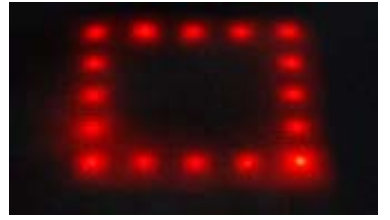

(b) Display of '0'
Figure 5. Fabric Input and Output Elements

\section{User Input and Output Element}

The user I/O devices are one of the most important devices because they are used directly by the user. Therefore, they should be easy to use, has high accessibility and comfortable to wear. The Fig.5 shows the example of the fabric input and output elements. The Fig.5(a) shows the capacitive type fabric touch sensor. The touch sensor uses the capacitance variation of the electrode. When the user touches the finger to the electrode, the parallel finger capacitance value $\left(\mathrm{C}_{\text {human }}\right)$ is added to the original electrode capacitance value $\left(\mathrm{C}_{0}\right)$. The read-out circuit detects the capacitance variation, and determines whether the finger touches the sensor. Fig.5(b) shows the LED array display which has $5 \times 5$ pixel resolution. The chip LEDs are soldered on P-FCB board by conductive adhesive. The array is controlled to display simple text or the number. Fig.5(b) shows he example display of ' 0 '.

\section{WeARABLE SySTEM IMPLEMENTATIONS}

The proposed P-FCB technique provides lots of possibility of the application. Among the many applications, we introduce two examples.
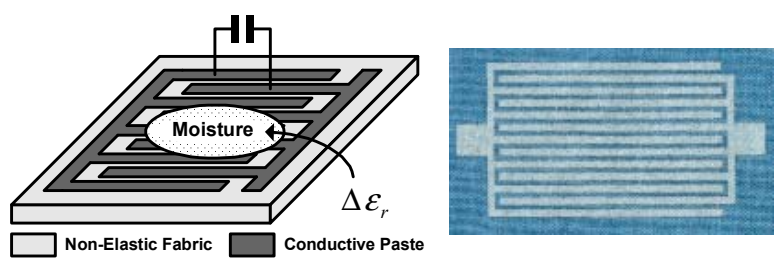

(a) Fabric Capacitor

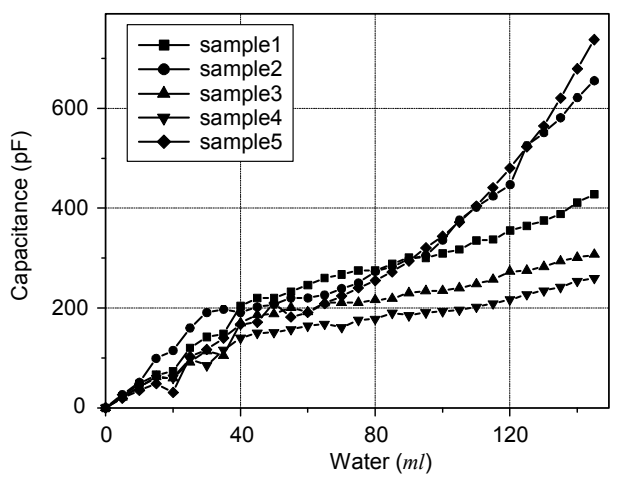

(b) Capacitance Variation

Figure 6. Fabric Capacitive Sensor by P-FCB 


\section{A. A Sweat Monitoring System}

The first one is the sweat monitoring system. The monitoring system sensor node consists of the fabric capacitance sensor, the monitoring processing module, and LED display are integrated on the clothes.

The fabric capacitor of Fig.6(a) is fabricated on $40 \mathrm{~mm} \times 25 \mathrm{~mm}$ fabric patch with interdigitated electrode of $1 \mathrm{~mm}$ line width and spacing. Its capacitance value can be changed by dielectric constant of the fabric, and the sensitivity can be modified by the number of interdigitated electrodes. The fabric contains pores inside and the humidity of the pores has a strong influence on the dielectric constant of the fabric [11]. Therefore, the fabric capacitor, which has $1 \mathrm{pF}$ capacitance value when it is dry, shows about $1 \mathrm{nF}$ capacitance value when a water drop is on the surface as shown in Fig.6(b). The overall range of capacitance values is from $1 \mathrm{pF}$ to $1 \mathrm{nF}$ which requires the capacitive sensor circuit with large dynamic range to cover it. The monitoring chip provides sensing, data processing and data transmission [1].

All measurements are conducted on wearable system as shown in Fig.7. When the humidity is detected by fabric sensor electrode, the monitoring chip senses the variation of the capacitance value and the make the LEDs to turn on. The detailed measured results are described by the number of pulses on the oscilloscope

\section{B. Fabric Antenna for RFID Tag}

The other example is the RFID antenna. The antenna is fabricated on the clothes directly, and the $900 \mathrm{MHz}$ RFID tag chip is bonded on the antenna as shown in Fig.8. The measurement of tag reading is executed by RFID reader kit. If the small size antenna is printed on the backside, the circuit system can be perfectly pervasive to the clothes.

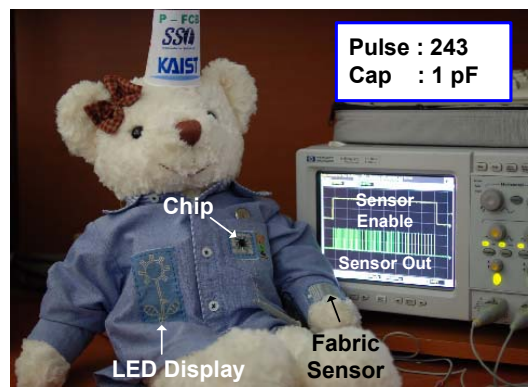

(a) LED Off without Water Drop

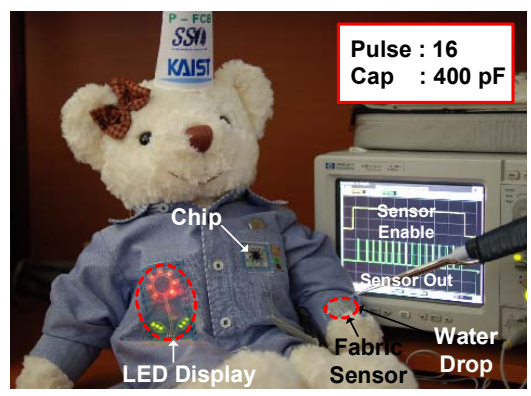

(b) LED Light-On with Water Drop on the Sensor

Figure 7. A Sweat Monitoring System Implemented on Clothes and Its Measurement Results

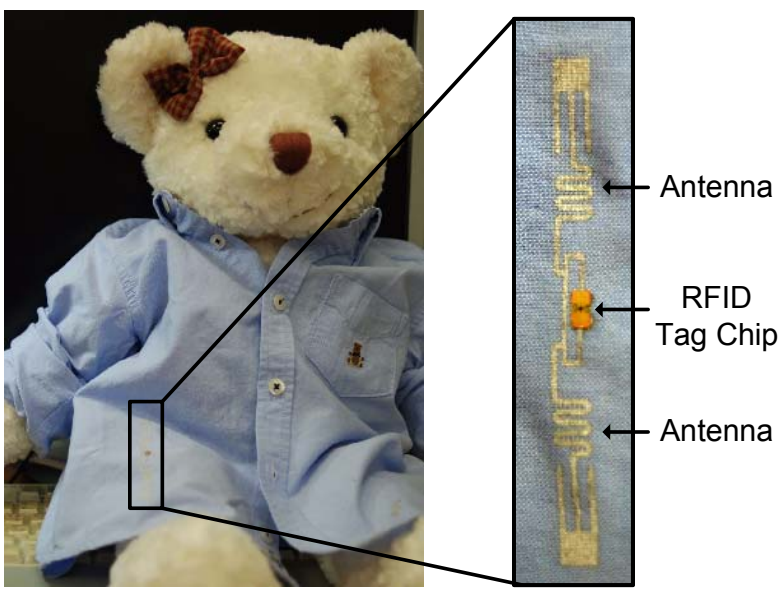

Figure 8. RFID Tag with Fabric Antenna

\section{CONCLUSION}

The planar-fashionable circuit board (P-FCB) technology is proposed which provides small size, light weight and high flexibility and wearability. The electrical elements such as passive elements, the fabric package, and the I/O devices can be fabricated by the P-FCB technology. Through the electrical and mechanical characteristic analysis of the PFCB, more efficient wearable system can be produced. The possibility of P-FCB technique is proved by real wearable system integration and measurement results.

\section{REFERENCES}

[1] H.Kim, "A 1.12mW Continuous Healthcare Monitor Chip Integrated on A Planar-Fashionable Circuit Board," Proc. of ISSCC, Feb.2008

[2] Y.Kim, "Electrical Characterization of Printed Circuits on the Fabric" IEEE Tran. Advanced Packaging (submitted)

[3] R.DeVaul, "MIThril 2003: Applications and Architecture", Proc. of ISWC, Oct.2003

[4] S.Park, "The wearable motherboard: a framework for personalized mobile information processing (PMIP)," Proc. of DAC, Jun. 2002

[5] E.Post, "E-broidery: Design and Fabrication of Textile-based Computing," IBM System Journal, vol.39, Nos.3\&4, pp.840-860, 2000

[6] S.Jung, "Enabling Technologies for Disappearing Electronics in Smart Textiles," Proc. of ISSCC, Feb. 2003

[7] T.Linz, "Embroidering Electrical Interconnects with Conductive Yarn for the Integration of Flexible Electronic Modules into Fabric," Proc. of ISWC, Oct. 2005

[8] L.Buechly, "Fabric PCBs, electronic sequins, and socket buttons: techniques for e-textile craft," Journal of Personal and Ubiquitous Computing, Jun. 2007

[9] D.Marculescu, "Electronic Textiles: A Platform for Pervasive Computing," Proc. of IEEE, Dec. 2003

[10] P.Holmes, "Handbook of thick film technology," Electrochemical Publications Ltd., 1976

[11] K. Ong, "Design and Application of a Wireless, Passive, Resonantcircuit Environmental Monitoring Sensor," Sensor and Actuators A Physical, vol.93, Issue 1, pp.33-43, Aug.2001 